\title{
Fine-Needle Aspiration of Breast Carcinomas With Prominent Lymphocytic Infiltrate
}

\author{
Celina G. Kleer, M.D., ${ }^{*}$ and Claire W. Michael, M.D.
}

Carcinomas of the breast with prominent lymphoplasmacytic background are commonly encountered in cytology. The aim of this study was to assess the prevalence of different types of carcinomas that share this common feature, identify possible distinguishing cytologic features, and evaluate the diagnostic pitfalls in this group of tumors. Eighteen fine-needle aspirations (FNAs) of breast carcinomas with heavy lymphoplasmacytic background were reviewed. Histologic follow-up was reviewed in all cases. Of 18 cases, there were 9 invasive ductal carcinomas (IDC), and 9 medullary carcinomas (6 typical and 3 atypical). FNAs from typical medullary carcinomas (TMC) showed more severe nuclear atypia and macronucleoli than the cases of IDC and atypical medullary carcinomas (AMC). Gland formation was absent in the $T M C$ but was common in IDC and AMC. No cytologic differences were noted between IDC and AMC. Nucleoli were larger in TMC (mean 4, $\mu \mathrm{m}$ ) than in AMC (mean, $2 \mu \mathrm{m}$ ) and IDC (mean, $1.5 \mu \mathrm{m}$ ). We conclude that lymphocytes and plasma cells may be seen in different types of breast carcinomas and should not be considered a diagnostic feature of TMC. Features potentially helpful in the cytologic differential diagnosis of a carcinoma with prominent lymphoplasmacytic background are nucleolar size (4 $\mu \mathrm{m}$ in $M C$, vs. 1.5 and $2 \mu \mathrm{m}$ in IDC and $A M C$, respectively) and the degree of nuclear atypia. Lymphocytosis may be part of the carcinoma or may originate from a lymph node involved by metastases. In rare cases, a prominent neutrophilic infiltrate may also be present. Diagn. Cytopathol. 2000;23:39-42. ๑ 2000 Wiley-Liss, Inc.

Key Words: breast; fine-needle aspiration; cytology; medullary carcinoma

Fine-needle aspiration (FNA) of breast carcinoma may contain a rich lymphoplamacytic infiltrate. While this feature has already been linked to typical medullary carcinoma (TMC), the prevalence of different variants of breast carcinoma in this group on FNA has not been previously evaluated.

Since TMC exhibits a lymphoplasmacytic background and high nuclear grade but carries a more favorable prognosis than

Department of Pathology, University of Michigan Medical Center, Ann Arbor, Michigan

*Correspondence to: Dr. Celina G. Kleer, Department of Pathology 2G332, University of Michigan Hospitals, 1500 E. Medical Center Drive, Ann Arbor, MI 48109-0054. E-mail: kleer@umich.edu

Received 21 September 1999; Accepted 14 January 2000 invasive ductal carcinoma, a specific diagnosis may be valuable in the preoperative adjuvant therapy of the patient.

This study was performed to assess the prevalence of the different breast carcinoma variants in this group and to identify any cytologic characteristics that may separate TMC from other variants. Very few reports have been published describing the cytological features of medullary carcinoma (MC) of the breast, ${ }^{1,2}$ and there are no studies to our knowledge that assess the differential cytologic features of typical MC, atypical MC, and invasive ductal carcinomas (IDC).

We present herein 18 malignant FNAs of the breast with histological follow-up that contained a lymphoplasmacytic background to assess the prevalence of different types of carcinomas that share this common cytologic feature and identify the cytologic features that separate TMC from other variants of breast carcinomas.

\section{Materials and Methods}

Eighteen cases of breast carcinoma were selected, based on the presence of prominent lymphoplasmacytic background, between 1986-1995. Hematoxylin-eosin-stained tissue sections from subsequent resection specimens were reviewed in all patients. The histologic diagnoses were based on strict and well-accepted criteria. ${ }^{3}$

Mammographically detected, nonpalpable masses were aspirated by a radiologist or surgeon under ultrasound guidance ( 8 cases), and palpable masses were aspirated by a cytopathologist (10 cases). Aspirators used 23- or 25-gauge needles attached to a 20-ml syringe with syringe holder (Aspir Gun, Linden, NJ). Aspirated material was expelled onto a glass slide and smeared using a second glass slide, spreading material evenly on the surfaces of both slides. The slides were immediately fixed in $95 \%$ ethanol and stained with the Papanicolau method. Cytologic features evaluated included cellularity, cellular arrangement, nuclear features, cytoplasmic features, and background cells.

In all cases, we measured the size of nucleoli at high power $(40 \times)$, using a micrometer. Five different areas in each smear were assessed, and the average was calculated. 
Table I. Clinical Summary of 18 Patients With Typical Medullary Carcinoma (TMC), Atypical Medullary Carcinoma (AMC), and Invasive Ductal Carcinoma (IDC) ${ }^{\mathrm{a}}$

\begin{tabular}{lcccc}
\hline Case no. & Age $(y r)$ & FNA site & $\begin{array}{c}\text { Cytologic } \\
\text { diagnosis }\end{array}$ & $\begin{array}{c}\text { Histologic } \\
\text { diagnosis }\end{array}$ \\
\hline 1 & 47 & Right & Pos ADC & TMC \\
2 & 52 & Right & Pos ADC & TMC \\
3 & 51 & Right & Pos ADC & TMC \\
4 & 59 & Right & Pos ADC & TMC \\
5 & 58 & Right & Pos ADC & TMC \\
6 & 45 & Left & Pos ADC & TMC \\
7 & 53 & Left & Pos ADC & AMC \\
8 & 37 & Left & Pos ADC & AMC \\
9 & 75 & Right & Pos ADC & AMC \\
10 & 51 & Right & Pos ADC & IDC \\
11 & 50 & Right & Pos ADC & IDC \\
12 & 47 & Right & Pos ADC & IDC \\
13 & 70 & Left & Pos ADC & IDC \\
14 & 74 & Right & Pos ADC & IDC \\
15 & 46 & Right & Pos ADC & IDC \\
16 & 70 & Right & Pos ADC & IDC \\
17 & 28 & Right & Pos ADC & IDC \\
18 & 32 & Left & Pos ADC & IDC \\
\hline
\end{tabular}

${ }^{\text {a } P o s, ~ p o s i t i v e . ~}$

Clinical information was obtained by reviewing patients' charts.

\section{Results}

The 18 cases of breast carcinoma with prominent lymphoplasmacytic background consisted of 9 IDC and 9 medullary carcinomas (6 TMC and $3 \mathrm{AMC}$ ). Table I summarizes the main clinico-pathologic findings of the 18 patients. Table II shows the different cytologic features observed in cases histologically confirmed as TMC, AMC, and IDC.

MC cases were markedly cellular and exhibited highly atypical cells arranged in syncytial fragments, numerous single cells, and bare nuclei (Figs. C1, C2). The cells had a high nuclear to cytoplasmic (N/C) ratio. The nuclear chromatin was coarse, and there was one or more macronucleoli (mean, $4 \mu \mathrm{m}$ ). Occasional cells were bizarre in shape (Fig. C-3). No gland formation was identified. Numerous polymorphonuclear leukocytes were seen in one case (Fig. C-4).

The IDC had varying degrees of cellular atypia, and some features overlapped with those of MC. The cells were mainly arranged as small aggregates and numerous single cells (Fig. C-5). Syncitial fragments were not common. Gland formation was commonly present (Fig. C-6). The cells had a high N/C ratio. The nuclei were hyperchromatic and contained variable-sized nucleoli (mean, $1.5 \mu \mathrm{m}$ ). No macronucleoli were noted. Finely vacuolated cytoplasm was frequently present.

Similarly, AMC showed a spectrum of cytological atypia (nucleoli size average was $2 \mu \mathrm{m}$ ), rendering them indistinguishable from IDC on cytologic smears.

We encountered two interesting cases. One such case was a well-circumscribed mass detected mammographically. FNA resulted in very cellular smears composed of markedly atypical cells with prominent nucleoli, in a background of numerous small lymphocytes and plasma cells (Fig. C-7). This case was thought to represent a TMC. When the mass was excised, metastatic high-grade IDC was present in an intramammary lymph node, with an occult primary. In another case, the tumor presented as a $3.5-\mathrm{cm}$ circumscribed mass in the right breast. FNA revealed scattered highly atypical cells in a background of numerous polymorphonuclear leukocytes, as well as lymphocytes (Fig. C-4). This picture raised the possibility of a breast abscess with atypical repair. Follow-up resection revealed TMC with a very prominent lymphoplasmatytic as well as neutrophilic infiltrate.

\section{Discussion}

The finding of a rich lymphoplasmacytic background in FNA from breast carcinomas is common, and often brings to mind the possibility of a medullary carcinoma. There is very little published on the cytologic features of this variant of invasive breast carcinoma, and we do not know whether the presence of numerous lymphocytes and plasma cells in the background of a very atypical neoplasm should point to a diagnosis of medullary carcinoma.

Typical medullary carcinoma (TMC) of the breast has been reported to carry a prognosis better than infiltrating ductal carcinoma, provided that strict histological criteria are used for the diagnosis.3,4,5 Studies have shown that the behavior of AMC and its prognosis do not differ significantly from those of IDC. 6,7

Previous studies indicated that TMCs are frequently overdiagnosed due to difficulty in diagnosis. ${ }^{8,9}$ A critical review of tumors initially diagnosed as TMC revealed a significant proportion to represent AMC or IDC. ${ }^{9}$ An overdiagnosis of TMC may result in undertreatment of poorly differentiated ductal carcinomas. Thus, strict criteria should be followed in making these diagnoses.

Histologically, definitive features required for the diagnosis of TMC include microscopic circumscription, syncy-

Table II. Comparison of Cytologic Features of Typical Medullary Carcinoma (TMC), Atypical Medullary Carcinoma, and Invasive Ductal Carcinoma

\begin{tabular}{|c|c|c|c|c|}
\hline & $\begin{array}{l}\text { Cellular smear with } \\
\text { syncytial fragments }\end{array}$ & Marked atypia & Macronиcleoli & $\begin{array}{l}\text { Vacuolization/ } \\
\text { gland formation }\end{array}$ \\
\hline Medullary cancer $(\mathrm{n}=6)$ & $5 / 6$ & $5 / 6$ & $6 / 6$ & $0 / 6$ \\
\hline Atypical medullary cancer $(\mathrm{n}=3)$ & $0 / 3$ & $3 / 3$ & $3 / 3$ & $1 / 3$ \\
\hline Invasive ductal cancer $(\mathrm{n}=9)$ & $2 / 9$ & $6 / 9$ & $3 / 9$ & $4 / 9$ \\
\hline
\end{tabular}



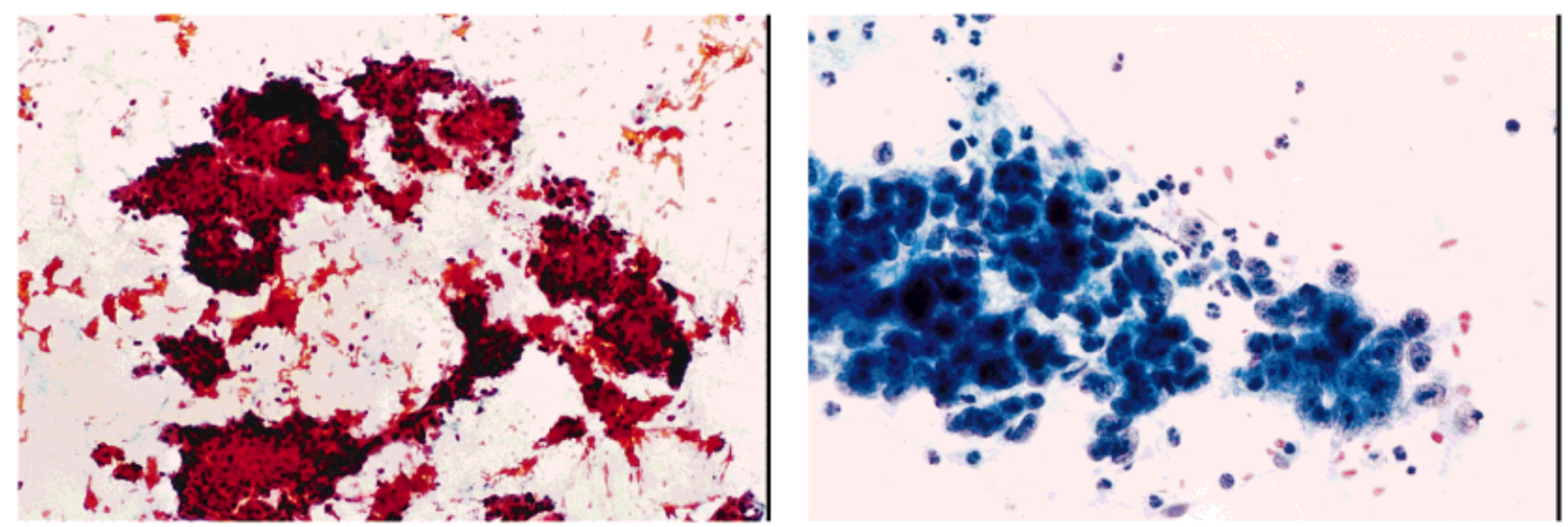

Fig. C-1

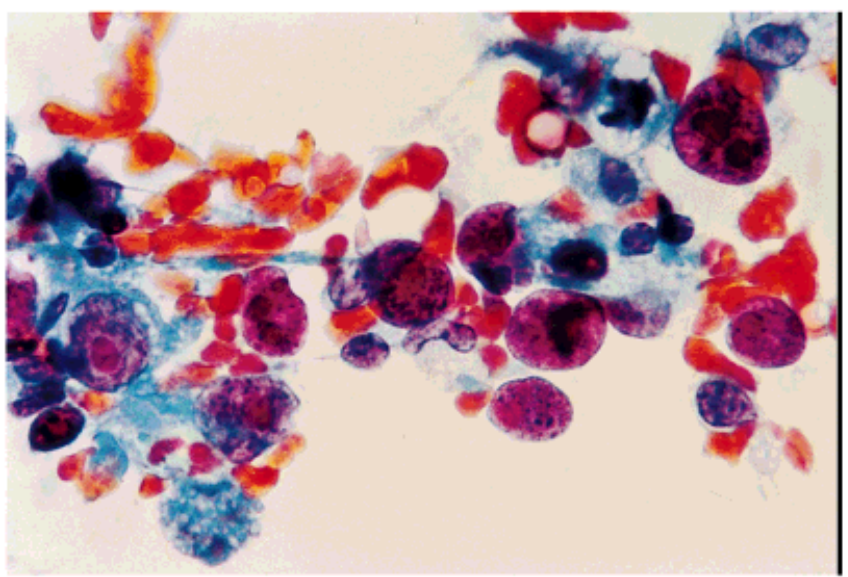

Fig. C-2

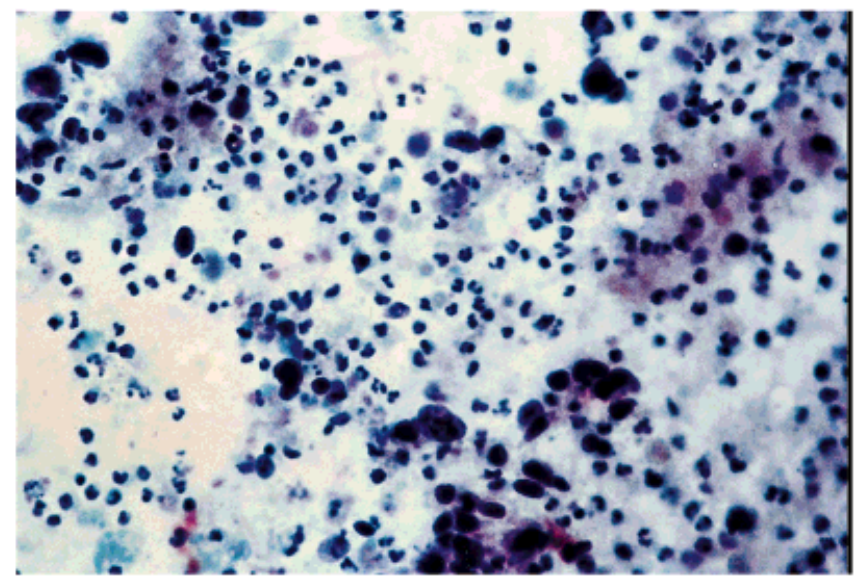

Fig. C-3

Fig. C-4

Fig. C-1. Typical medullary carcinoma. Very cellular smear, with syncytial fragments and hemorrhage (Papanicolaou stain, $\times 40)$. Fig. C-2. Typical medullary carcinoma, with syncytial fragment and markedly atypical cells. Note inflammatory cells in the background (Papanicolaou stain, $\times$ 200). Fig. C-3. Typical medullary carcinoma, with bizarre cells with bare nuclei and macronucleoli (Papanicolaou stain. Oil, $\times$ 600). Fig. C-4. Numerous polymorphonuclear leukocytes obscuring the neoplastic cells in a case of typical medullary carcinoma (Papanicolaou stain, $\times 100$ ).

tial pattern of growth, poorly differentiated nuclear features with high mitotic rate, and intense lymphoplasmacytic reaction. When most but not all of these features are present, the tumor may be diagnosed as AMC. $3,5,10,11$

There is little in the literature describing the cytological features of TMC. Classic descriptions emphasize the presence of numerous malignant cells arranged in clusters and syncytial groupings, with numerous lymphocytes in the background. ${ }^{1,2}$ Cytological features of AMC have not been reported to date.

Cytological features of IDC are well-described in the literature. ${ }^{1,2,12-14}$ These include cellular smears with threedimensional clusters exhibiting discohesion and numerous loosely cohesive single malignant cells. There are no bipolar naked nuclei, and there is tumor diathesis.

In the present study, we found that numerous lymphocytes and plasma cells are seen in IDC, TMC, and AMC. Despite significant overlap in the cytologic characteristics among these neoplasms, features potentially helpful in the differential diagnosis of a carcinoma with prominent lymphoplasmacytic background are nucleolar size $(4 \mu \mathrm{m}$ in TMC, vs. 1.5 and $2 \mu \mathrm{m}$ in IDC and AMC, respectively), and the degree of nuclear atypia.

The two interesting cases presented above, a metastatic high-grade IDC to an intramammary lymph node with an occult primary and a TMC with malignant cells obscured by a prominent neutrophilic infiltrate suggestive of a breast abscess, illustrate limitations of FNA in the diagnosis of TMC.

In summary, several variants of breast carcinoma may contain a prominent lymphocytic infiltrate on FNA. Although it may not be very accurate by cytologic evaluation, the recognition and differentiation of TMC from AMC and IDC are clinically relevant. The only potentially helpful features in the cytologic differential diagnosis are nucleolar size $(4 \mu \mathrm{m}$ in MC, vs. 1.5 and $2 \mu \mathrm{m}$ in IDC and AMC, respectively), and degree of nuclear atypia. 


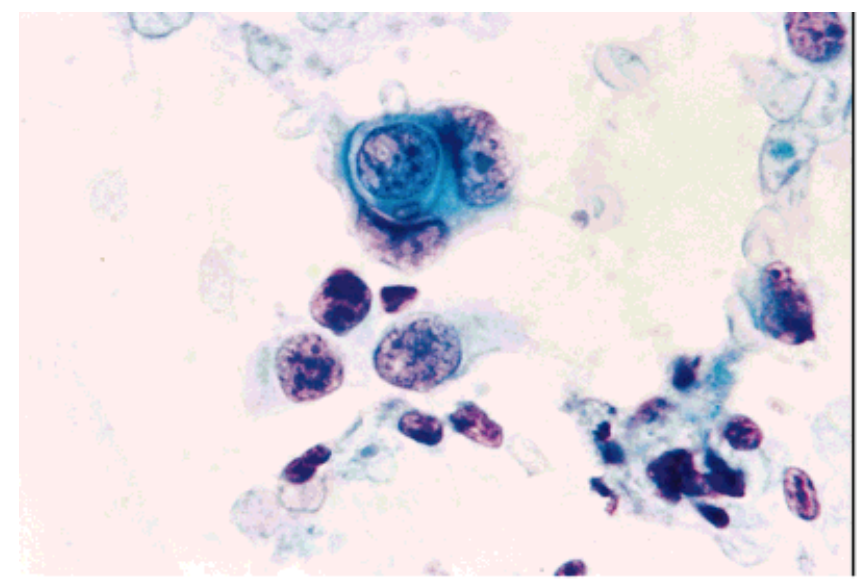

Fig. C-5

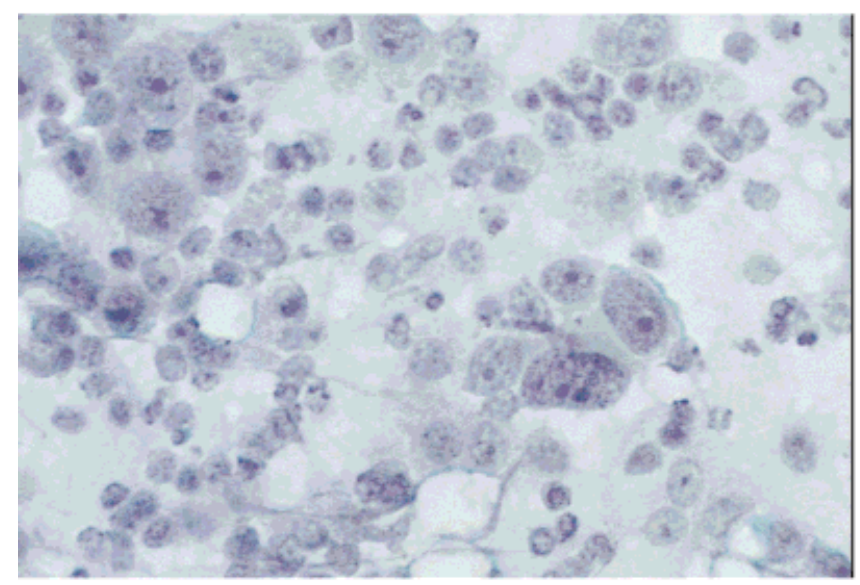

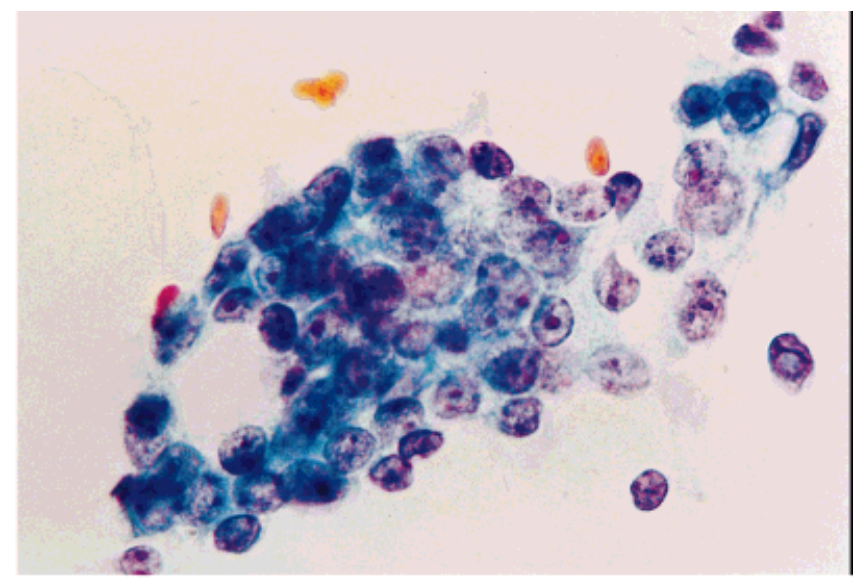

Fig. C-6

Fig. C-7

Fig. C-5. Invasive ductal carcinoma with small aggregate of neoplastic cells, single cells, and scattered lymphocytes (Papanicolaou stain. Oil, $\times 600$ ). Fig. C-6. Gland formation in invasive ductal carcinoma. Also note the finely vacuolated cytoplasm of the cells $($ Papanicolaou stain, $\times 400)$. Fig. C-7. Very cellular smear with markedly atypical cells in a background of lymphocytes corresponding to metastatic adenocarcinoma to an intramammary lymph node (Papanicolaou stain, $\times 400$ ).

\section{References}

1. Bibbo M. Medullary carcinoma of the breast. In: Bibbo M, editor. Comprehensive cytopathology. Philadelphia: W.B. Saunders; 1991. p 732-734.

2. De May RM. Medullary carcinoma of the breast. In: De May RM, editor. The art and science of cytopathology. Volume 2. Chicago: ASCP Press; 1996. p 876-877.

3. Ridolfi RL, Rosen PP, Port A, Kinne D, Mike V. Medullary carcinoma of the breast. A clinicopathologic study with 10 year follow-up. Cancer 1977;40:1365-1385.

4. Lidang Jensen M, Kiaer H, Andersen J, Jensen V, Melsen F. Prognostic comparison of three classifications for medullary carcinomas of the breast. Histopathology 1997;30:523-532.

5. Moore OS, Foote FW. The relatively favorable prognosis of medullary carcinoma of the breast. Cancer 1949;2:635-642.

6. Maier WP, Rosemond GP, Goldman LI, Kaplan GF, Tyson RR. A ten year study of medullary carcinoma of the breast. Surg Gynecol Obstet 1977;144:695-698.

7. Black CL, Morris DM, Goldman LI, McDonald JD. The significance of lymph node involvement in patients with medullary carcinoma of the breast. Surg Gynecol Obstet 1983;157:497-499.

8. Wargotz ES, Silverberg SG. Medullary carcinoma of the breast: a clinicopathologic study with appraisal of current diagnostic criteria. Hum Pathol 1988;19:1340-1346.
9. Rubens JR, Lewandrowski KV, Kopans DB, Koerner FC, Hall DA, McCarthy KA. Medullary carcinoma of the breast. Overdiagnosis of a prognostically favorable neoplasm. Arch Surg 1990;125:601-604.

10. Foote FW Jr, Stewart FW. A histologic classification of carcinoma of the breast. Surgery 1946;19:74-99.

11. Rosen PP. Medullary carcinoma. In: Rosen PP, editor. Rosen's breast pathology. Phil Lippincott-Raven, Philadelphia, PA. 1996:355-373.

12. Teixidor HS, Wojtasek DA, Reiches EM, et al. Fine-needle aspiration of breast biopsy specimens: correlation of histologic and cytologic findings. Radiology 1992;184:55-58.

13. Pennes DR, Naylor B, Rebner M. Fine needle aspiration biopsy of the breast: influence of the number of passes and the sample size on the diagnostic yield. Acta Cytol 1990;34:673-676.

14. Wang HH, Ducatman BS, Eick D. Comparative features of ductal carcinoma in situ and infiltrating ductal carcinoma of the breast on fine-needle aspiration biopsy. Am J Clin Pathol 1989;92:736-740.

15. Tavassoli FA. Infiltrating carcinomas, common and familiar special types: medullary carcinoma. In: Pathology of the breast. Norwalk, CT: Appleton \& Lange; 1992. p 333-339.

16. Fisher ER, Kenny JP, Sass R, et al. Medullary cancer of the breast revisited. Breast Cancer Res Treat 1990;16:215-229. 